\title{
Nanostructured $\mathrm{MnGa}$ films on $\mathrm{Si} / \mathrm{SiO}_{2}$ with $20.5 \mathrm{kOe}$ room temperature coercivity
}

\author{
C. L. Zha, ${ }^{1,2, a)}$ R. K. Dumas, ${ }^{3}$ J. W. Lau, ${ }^{4}$ S. M. Mohseni, ${ }^{1}$ Sohrab R. Sani, ${ }^{1}$ \\ I. V. Golosovsky, ${ }^{5}$ A. F. Monsen, ${ }^{2}$ J. Nogués, ${ }^{1,6,7}$ and Johan Åkerman ${ }^{1,3, b)}$ \\ ${ }^{1}$ Materials Physics, Royal Institute of Technology (KTH), Electrum 229, 16440 Kista, Sweden \\ ${ }^{2}$ Department of Physics, Norwegian University of Science and Technology (NTNU), 7491 Trondheim, Norway \\ ${ }^{3}$ Department of Physics, University of Gothenburg, 41296 Gothenburg, Sweden \\ ${ }^{4}$ Metallurgy Division, National Institute of Standards and Technology, Gaithersburg, Maryland 20899, USA \\ ${ }^{5}$ St. Petersburg Nuclear Physics Institute, 188300 Gatchina, St. Petersburg, Russia \\ ${ }^{6}$ Institució Catalana de Recerca i Estudis Avançats (ICREA), Barcelona, Spain \\ ${ }^{7}$ CIN2(ICN-CSIC), Universitat Autònoma de Barcelona, Catalan Institute of Nanotechnology, \\ Campus de la UAB, 08193 Bellaterra (Barcelona), Spain
}

(Received 9 May 2011; accepted 22 September 2011; published online 1 November 2011)

\begin{abstract}
Nanostructured $\mathrm{Mn}_{67} \mathrm{Ga}_{33}$ films exhibiting high room temperature coercivity $\left(H_{C}=20.5 \mathrm{kOe}\right)$ have been prepared by sputtering onto thermally oxidized Si substrates. Both the morphology and the coercivity of the films can be tuned by varying the growth parameters. The low deposition rate film, sputtered at a reduced power and working pressure, demonstrates a discontinuous island-like growth and the highest $H_{C}$. The large $H_{C}$ is linked to the presence of the high anisotropy $\mathrm{DO}_{22}$ $\mathrm{Mn}_{3} \mathrm{Ga}$ phase and the single domain character of the exchange isolated, dipolar interacting, single crystal islands. (C) 2011 American Institute of Physics. [doi:10.1063/1.3656457]
\end{abstract}

\section{INTRODUCTION}

Magnetic thin films and nanostructured magnetic materials exhibiting a high room temperature coercivity have attracted considerable attention for permanent magnet applications, ${ }^{1}$ nano- and micro-electromechanical systems (NEMS-MEMS), ${ }^{2}$ new types of recording media, ${ }^{3}$ and as reference materials in pseudo-spin valves (e.g., in magnetoresistive random access memory (MRAM)). ${ }^{4}$ Apart from classical permanent magnetic materials such as Co-based alloys (e.g., $\mathrm{Co}_{3} \mathrm{Pt}, \mathrm{CoCrPtX}$, etc.), $L 1_{0}$ alloys (e.g., FePt, MnAl, CoPt, $\mathrm{FePd}$, etc.), and rare earth-transition metal alloys (e.g., $\left.\mathrm{SmCo}_{5}, \mathrm{Nd}_{2} \mathrm{Fe}_{14} \mathrm{~B}\right){ }^{5}$ novel alternative materials such as $\varepsilon-\mathrm{Fe}_{2} \mathrm{O}_{3},{ }^{6}$ hexagonal $\mathrm{MnBi},{ }^{7}$ and $\mathrm{DO}_{22} \mathrm{Mn}_{2-3} \mathrm{Ga}$ (Ref. 8) are currently being investigated. $\mathrm{DO}_{22} \mathrm{Mn}_{2-3} \mathrm{Ga}$ has recently garnered particular interest since it was theoretically predicted to be a half-metallic-like ferrimagnet with $88 \%$ spin polarization $^{9}$ and experimentally shown to exhibit spin polarization as high as 58\%. ${ }^{10}$ Moreover, it has been reported to exhibit very low damping constant. ${ }^{11}$ Therefore, it has been proposed to have great potential for applications in spin transfer-torque devices, ${ }^{9}$ such as spin-transfer-torque MRAM, ${ }^{12}$ and spin torque nano-oscillators. ${ }^{13}$

Bulk $\mathrm{DO}_{22} \mathrm{Mn}_{2-3} \mathrm{Ga}$ has been reported to have a large coercivity, $H_{C}$, and moderate saturation magnetization, $M_{S}$, of $13.5 \mathrm{kOe}$ and $300 \mathrm{emu} / \mathrm{cm}^{3}$, respectively. ${ }^{8}$ Somewhat smaller values have been recently reported by Winterlik et al. $\left(H_{C}=5.7 \mathrm{kOe}, M_{S} \sim 190 \mathrm{emu} / \mathrm{cm}^{3}\right) .{ }^{14}$ By using pseudoepitaxial growth on single crystal substrates, Wu et al. were able to achieve a high chemical ordering degree of the $\mathrm{DO}_{22}$ phase with a giant magnetocrystalline anisotropy of $K_{\text {eff }}$ $\sim 1.2 \times 10^{7} \mathrm{erg} / \mathrm{cm}^{3}$. The resulting thin films exhibited a

\footnotetext{
${ }^{a)}$ Electronic mail: chaolinzha@gmail.com.

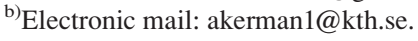

comparable coercivity and magnetization $\left(H_{C} \sim 6.6 \mathrm{kOe}, M_{S}\right.$ $\sim 250 \mathrm{emu} / \mathrm{cm}^{3}$ ) (Ref. 15) to the bulk values. However, Kurt et al. have reported epitaxial films with large $H_{C}$ but somewhat low $M_{S}\left(H_{C} \sim 19.0 \mathrm{kOe}, M_{S} \sim 110 \mathrm{erg} / \mathrm{cm}^{3}\right) .{ }^{10}$ Moreover, Wu et al. also reported electrical transport properties of perpendicularly magnetized $\mathrm{DO}_{22} \mathrm{MnGa}$ epitaxial films, consistent with a high spin polarization. ${ }^{16}$ In our previous study we have demonstrated the utility in using $\mathrm{DO}_{22} \mathrm{MnGa}$ as a reference layer in pseudo spin valves. ${ }^{17}$ Although progress has been made in bulk and thin films, little is known about the behavior of nanostructured $\mathrm{DO}_{22} \mathrm{MnGa}$.

In this paper we report on the growth of nanostructured, discontinuous, $\mathrm{Mn}_{67} \mathrm{Ga}_{33}$ thin films containing the ferrimagnetic $\mathrm{DO}_{22} \mathrm{Mn}_{3} \mathrm{Ga}$ phase by magnetron sputtering on inexpensive, thermally oxidized, Si substrates. After proper tuning of the sputtering parameters a record high room temperature coercivity, $H_{C}=20.5 \mathrm{kOe}$, and moderate saturation magnetization, $M_{S}=140 \mathrm{emu} / \mathrm{cm}^{3}$, have been achieved. As the temperature is lowered the coercivity further increases to $H_{C}=28.2 \mathrm{kOe}$ at $5 \mathrm{~K}$. Nanostructuring, by properly tuning the growth conditions, is therefore a simple approach to significantly enhance the coercivity in these materials.

\section{EXPERIMENTAL}

All film stacks were deposited on thermally oxidized $\mathrm{Si}$ (100) substrates using a magnetron sputtering system with base pressure of better than $5 \times 10^{-8}$ Torr. The $200 \mathrm{~nm}$ thick MnGa films were sputtered using a $\mathrm{Mn}_{60} \mathrm{Ga}_{40}$ alloy target. During deposition the substrates were held at $400{ }^{\circ} \mathrm{C}$ and subsequently annealed in situ at the same temperature for an additional $15 \mathrm{~min}$. Two different growth rates are presented here. The high-rate $(0.11 \mathrm{~nm} / \mathrm{s})$ sample is deposited at a DC power of $75 \mathrm{~W}$ and Ar working pressure of 5.0 mTorr while the low-rate $(0.08 \mathrm{~nm} / \mathrm{s})$ sample was deposited at $30 \mathrm{~W}$ and 
$1.5 \mathrm{mTorr}$. Ta $(6 \mathrm{~nm})$ buffer and capping layers were deposited at room temperature to provide a fresh growth surface for the MnGa film and to prevent oxidation of its top surface, respectively.

Top and cross-section views of the microstructure were studied using high-resolution field-emission scanning electron microscopy (HR-SEM) and high-resolution field-emission transmission electron microscopy (HR-TEM). Electron energy-loss spectra (EELS) acquired in scanning TEM (STEM) mode provided the local Ga and Mn distribution. The global composition of the films were characterized by energy dispersive $\mathrm{x}$-ray spectrometry and found to be $\mathrm{Mn}_{67} \mathrm{Ga}_{33}$, i.e., within the 66-74 at.\% Mn content range required to achieve the $\mathrm{DO}_{22}$ phase. ${ }^{8}$ The surface topography was studied using atomic force microscopy (AFM). Crystallographic structures were investigated by $\mathrm{x}$-ray diffraction (XRD) using $\mathrm{Cu} \mathrm{K} \alpha$ radiation in symmetric scan geometry. The top $100 \mathrm{~nm}$ of the films were studied by glancing incidence XRD (GIXRD) with a fixed incident angle of $0.85^{\circ}$. The XRD and GIXRD patterns were analyzed using the FullProf code. ${ }^{18}$ Magnetic properties were characterized using vibrating sample magnetometer (VSM) with a maximum field of $90 \mathrm{kOe}$. The magnetic domain structure was observed using a magnetic force microscope (MFM).

\section{RESULTS AND DISCUSSION}

The XRD patterns (Fig. 1) for the two MnGa films are similar and show the multiphase nature of both samples. The Rietveld refinement of the patterns reveals the presence of two phases, $\beta-\mathrm{Mn}(\mathrm{Ga})$ with a cubic $\mathrm{P}_{1} 32$ structure and stoichiometric $\mathrm{DO}_{22} \mathrm{Mn}_{3} \mathrm{Ga}$ with a tetragonal I4/mmm structure. The volume ratio of both phases depends on the deposition rate with $12(1) \%$ of $\mathrm{DO}_{22}$ phase for the low rate sample and $14(1) \%$ for the high rate one. The lattice parameters for the $\mathrm{DO}_{22}$ phase are $\mathrm{a}=0.38994(6) \mathrm{nm}$ and $\mathrm{c}=0.7116(2) \mathrm{nm}$ with $\mathrm{c} / \mathrm{a}=1.8248$ for the low rate sample and $\mathrm{a}=0.39032(4)$ $\mathrm{nm}$ and $\mathrm{c}=0.7142(2) \mathrm{nm}$ with $\mathrm{c} / \mathrm{a}=1.8300$ for the high rate sample, respectively. The lattice parameters and the c/a

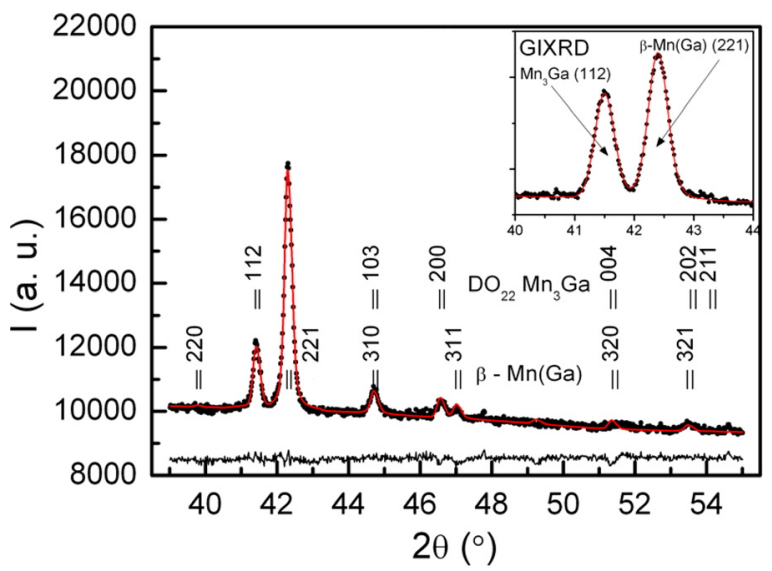

FIG. 1. (Color online) XRD pattern of the low-rate $(0.08 \mathrm{~nm} / \mathrm{s}) \mathrm{MnGa}$ film, where the symbols are the experimental data and the thick line is the calculated curve. The thin line below the main pattern is the difference between the observed and calculated patterns. Shown in the inset is the GIXRD pattern for the same sample. The vertical stripes mark the position of the $\beta$ - $\mathrm{Mn}(\mathrm{Ga})$ and $\mathrm{DO}_{22} \mathrm{Mn}_{3} \mathrm{Ga}$ Bragg reflections. ratios for the $\mathrm{DO}_{22} \mathrm{Mn}_{3} \mathrm{Ga}$ phase are consistent with literature values. ${ }^{8,14,15}$ The crystallite sizes, D, were found to be $\mathrm{D}=49.1(1.7)$ and $\mathrm{D}=41.7(1.1) \mathrm{nm}$, for the low and high rate samples. Interestingly, the GIXRD results show a relative increase of the $\mathrm{DO}_{22}$ (112) peak with respect to the $\beta$-Mn $(\mathrm{Ga})$ (221) one (Fig. 1 inset), indicating that the amount of $\mathrm{DO}_{22}$ phase dramatically increases to $88(1) \%$ toward the surface, as confirmed by the Rietveld refinement.

The top-view SEM images show that the films do not grow homogeneously but have nanostructured island-like morphology (Figs. 2(a) and 2(b)). Both the top- and crosssection views (Figs. 2(a) and 2(c)) indicate that the islands in the high-rate film are actually highly interconnected. On the other hand, the low-rate film (Figs. 2(b) and 2(d)) is composed of independent, discontinuous islands with a columnar-like structure with diameters of hundreds of nanometers. The AFM images (Figs. 3(a) and 3(b)) corroborate this picture. Although the overall morphology is similar for both samples (Figs. 3(a) and 3(b)), the root-mean square (rms) roughness (over $3 \times 3 \mu \mathrm{m}^{2}$ ) of the low-rate sample, 39.7(3) $\mathrm{nm}$, is larger than 33.6(2) nm rms-roughness of the high-rate sample. Similarly, as can be seen in the line scans the peak-to-valley values in Figs. 3(c) and 3(d) are much larger for the low-rate sample.
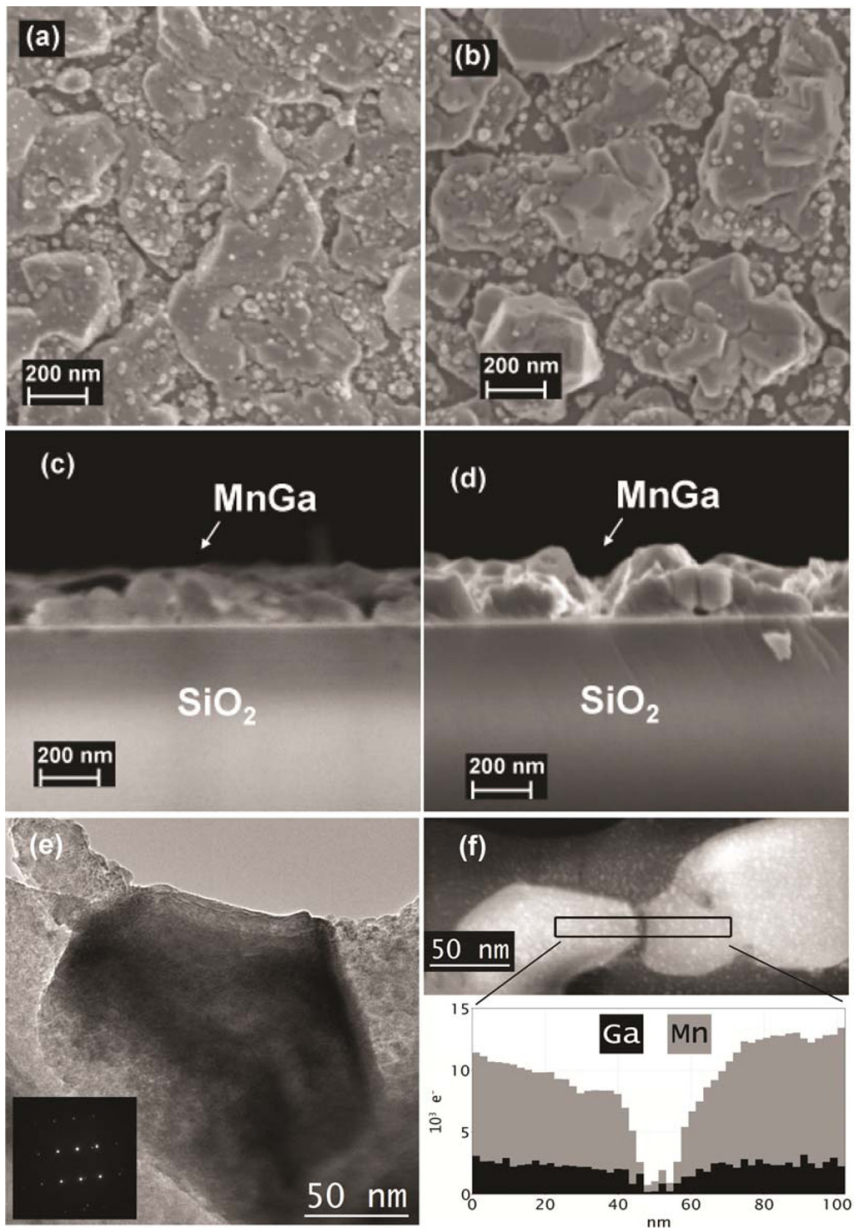

FIG. 2. (Color online) (a), (b) Top view and (c), (d) cross-sectional view SEM images of the high-rate (left) and low-rate (right) MnGa films, respectively. (e) Plane-view bright-field HR-TEM image and (f) STEM image (top) and chemical profile obtained from electron energy-loss spectroscopy (bottom) of the low-rate sample. The inset in (e) is a selected area electron diffraction pattern. 

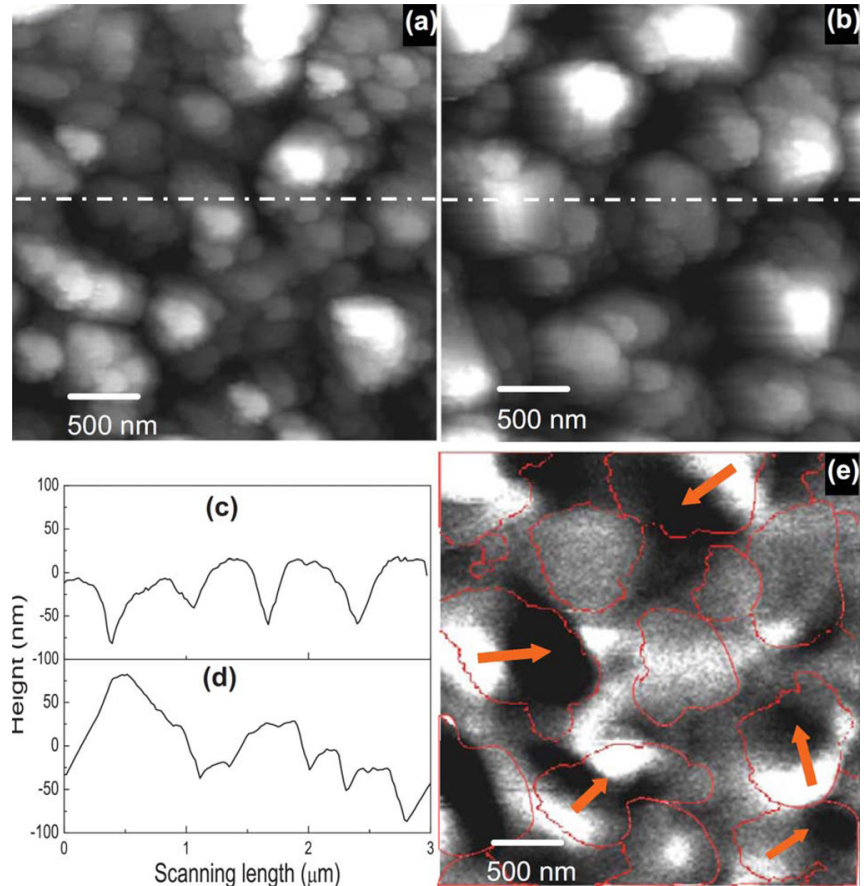

FIG. 3. (Color online) AFM image $\left(3 \times 3 \mu \mathrm{m}^{2}\right.$ scan area) and line scans (white dashed lines) for the (a), (c) high-rate and (b), (d) low-rate MnGa films. (e) MFM image of the low-rate MnGa film where the island from (b) are outlined for guidance. The arrows indicate the single domain contrast with an in-plane component found in many of the islands. Measurements were carried out on thermally demagnetized samples.

The roughness exponent of the two samples [0.62(1) -low rate- and 0.70(1) -high rate-] further confirms the rougher character of the low rate films. ${ }^{19,20}$ This implies that in the low-rate sample the islands are more isolated and consequently the tip can penetrate deeper in between the islands. The high-rate film is more prone to form a continuous film due to the higher kinetic energy and mobility of the sputtered atoms. However, the low-rate films, which lack the necessary kinetic energy, tend to accumulate and form the island-like structure. $^{21}$

The microstructure of the low-rate sample was also investigated by TEM. Figure 2(e) is a bright-field image showing a typical island. Though not evident from this image, lattice fringes may be seen from thinner parts of such islands at higher magnifications. Selected area electron diffraction showed that these islands are predominately single crystals. The inset of Fig. 2(e) is a diffraction pattern from one such island. The top of Fig. 2(f) is a STEM image of two well separated islands. The rectangular box in this image indicates a segment where chemical distribution of $\mathrm{Mn}$ and $\mathrm{Ga}$ was mapped with electron energy-loss spectra. Using the $\mathrm{L}_{2-3}$ edge of $\mathrm{Mn}$ and $\mathrm{Ga}$, we generated the chemical profile of these two elements along the length of the rectangular box, which is showed in the bottom of Fig. 2(f). Clearly, the concentration of $\mathrm{Ga}$ and $\mathrm{Mn}$ drops off rapidly as the electron beam scans near the island boundaries and the trench in between.

Hysteresis loops measured both parallel and perpendicular to the film plane are shown in Figs. 4(a) and 4(b) for the high- and low-rate films, respectively. Remarkably, the inplane and out-of-plane hysteresis loops are virtually identical in both samples, suggesting a random orientation of the easy

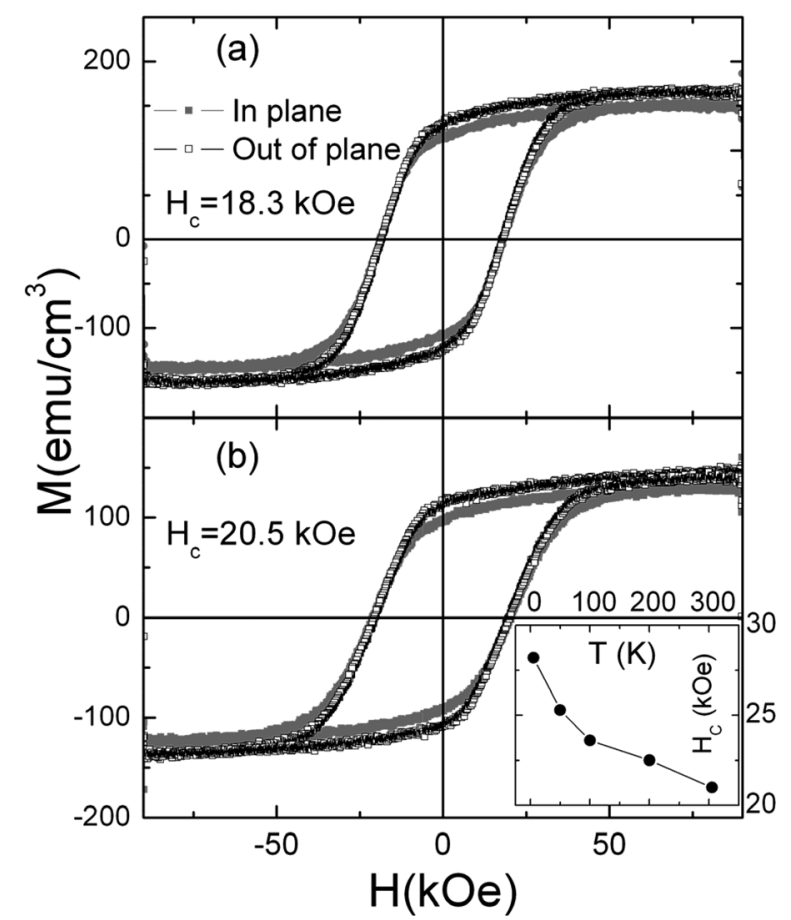

FIG. 4. In-plane and out-of-plane hysteresis loops for (a) high-rate and (b) low-rate MnGa films. Inset in (b) shows the temperature dependence of the coercivity of the low-rate sample.

magnetic axis in both films. Note that the rather high squareness of the loops, compared to $\mathrm{M}_{\mathrm{R}} / \mathrm{M}_{\mathrm{S}}=0.5$ for randomly oriented uniaxial single domain particles, ${ }^{22}$ implies some degree of dipolar coupling between the islands. The highrate film has $H_{C}=18.3 \mathrm{kOe}$ and $M_{S}=151 \mathrm{emu} / \mathrm{cm}^{3}$, respectively. The $H_{C}$ of the low-rate sample is found to increase to $20.5 \mathrm{kOe}$ while a small drop in $M_{S}, M_{S}=140 \mathrm{emu} / \mathrm{cm}^{3}$, is also observed. As the temperature is lowered $H_{C}$ of the lowrate sample further increases to $28.2 \mathrm{kOe}$ (see inset in Fig. 4(b)). Importantly, it should be emphasized that this may not be the intrinsic maximum $\mathrm{H}_{\mathrm{C}}$ for this material, since the considerable anisotropy field, $\mathrm{H}_{\mathrm{K}}=2 \mathrm{~K}_{\mathrm{u}} / \mathrm{M}_{\mathrm{S}} \sim 96 \mathrm{kOe}$ (calculated using $\mathrm{Wu}$ et al. data ${ }^{15}$ ), for this material certainly leaves room for even larger coercivities.

The moderate $M_{S}$ observed in our films is consistent with previously reported values ${ }^{8,14,15}$ and the multiphase character of our samples. The large coercivity of both samples is primarily linked to the presence of the high anisotropy $\mathrm{DO}_{22} \mathrm{Mn}_{3} \mathrm{Ga}$ phase although slightly lower degree of ordering in our samples may imply a somewhat smaller anisotropy. Therefore, the increased coercivity of the low-rate sample over the high-rate sample is ascribed to the more pronounced island-like morphology of the films, as observed for other systems. ${ }^{23}$ Nevertheless, given the multiphase-polycrystalline nature of our films, other sources for the different coercivity between the high-rate and low-rate samples, such as small differences in stoichiometry or ordering parameter, cannot be ruled out.

The impact of the sample morphology on the enhanced coercivity can be explained by its influence on the magnetic domain structure and the likelihood of magnetic domain wall propagation. In the low-rate sample, each island is magnetically isolated from all other islands, and the global magnetic 
properties of the film correspond to the sum of all individual islands. We can estimate the critical size, $d_{C}$, for single domain behavior using $d_{C}=24 \sqrt{A K_{u}} / N M_{S}^{2},{ }^{24}$ where $K_{u}, A$, and $N$ are the uniaxial anisotropy, the exchange stiffness constant, and the demagnetization factor, respectively. Using $K_{u}=1.2 \times 10^{7} \mathrm{erg} / \mathrm{cm}^{3}, M_{S} \sim 250 \mathrm{emu} / \mathrm{cm}^{3}$, in $\mathrm{DO}_{22} \mathrm{MnGa}$ from Wu et al.,$^{15}$ and assuming $A=1 \times 10^{-6} \mathrm{erg} / \mathrm{cm}$ and $N$ $\sim 4 \pi / 3$, leads to $d_{C} \sim 3.2 \mu \mathrm{m}$. While this calculation is only a rough estimation, since $d_{C}$ is dominated by $M_{S}{ }^{-2}$ and we use $M_{S}$ values among the highest reported in the literature, we are confident that the observed island size of only a few hundred $\mathrm{nm}$ is certainly smaller than any reasonable $d_{C}$ for this material. Indeed, the MFM image of the low-rate sample clearly confirms the single domain character of the islands (Fig. 3(e)). The islands, from Fig. 3(b), are outlined in the MFM image and the arrows indicate some of the single domain structures which exhibit strong black/white contrast typical of a magnetic dipole. From the MFM and GIXRD results we can infer that the $\mathrm{DO}_{22} \mathrm{Mn}_{3} \mathrm{Ga}$ should be on the top part of the islands. In the high-rate sample the islands are more interconnected and thus may be larger than $d_{C}$. This leads to multidomain structures and reversal by domain propagation, and consequently a reduction in $\mathrm{H}_{C}{ }^{25}$ However, the still considerably large coercivity of the high-rate sample can be explained by the rough microstructure, grain boundaries, and other imperfections (e.g., nonmagnetic phases) which inevitably lead to considerable domain wall pinning. ${ }^{26}$

As is typically found in hard magnetic systems, ${ }^{1,5}$ the presence of non-magnetic or weakly magnetic phases is crucial for both strong domain wall pinning and magnetic separation of individual grains, both of which contribute to a large coercivity. Unfortunately, there is usually a trade-off between high coercivity and high saturation magnetization in such systems. The specific energy product $E=H_{C} \times B_{r}$, where $B_{r}$ is the remanence, can be used as a figure of merit for permanent magnets where both a high $H_{C}$ and remanence are desired. ${ }^{14}$ In analyzing data from the literature we find a maximum $E$ of 25 and 20 MGOe for bulk ${ }^{8}$ and thin film ${ }^{15}$ $\mathrm{DO}_{22} \mathrm{MnGa}$ material systems, respectively. Using nanostructuring as a morphology based approach to decouple grains into single domains, we simultaneously achieve high coercivity and a substantial saturation magnetization. The 30 MGOe energy product realized in our low-rate film is $20 \%$ larger than that reported in bulk and 50\% larger than that previously reported in thin films.

\section{CONCLUSIONS}

In summary, by properly adjusting the sputtering parameters we have demonstrated nanostructured $\mathrm{MnGa}$ films on $\mathrm{Si} / \mathrm{SiO}_{2}$ with record high room temperature coercivity in excess of $20 \mathrm{kOe}$ without a significant reduction in the saturation magnetization. Consequently, the energy product $E$ is improved compared to the previously reported values. The high coercivity and high energy product of our nanostructured $\mathrm{MnGa}$ films are attributed to both the presence of the high anisotropy $\mathrm{DO}_{22} \mathrm{Mn}_{2-3} \mathrm{Ga}$ phase and the magnetic single domain character of the isolated, exchange-decoupled single crystal islands (interacting through dipolar forces).

\section{ACKNOWLEDGMENTS}

C.Z. thanks Dr. Erik Wahlstrøm for the critical comments on this manuscript. We are grateful to S. Lidin for giving us access to the PPMS. Support from The Swedish Foundation for strategic Research (SSF), The Swedish Research Council (VR), the Göran Gustafsson Foundation, and the Knut and Alice Wallenberg Foundation is gratefully acknowledged. J.N. would like to thank the Catalan DGR (2009SGR1292), and the Spanish MICINN (MAT2010-20616-C02) projects for partial financial support. I.V.G. thanks the EU ONDA project (FP7-PEOPLE-2009-IRSES-247518), the Russian RFBR 10-02-00576 and MESRF 16.518.11.7034 grants and the Generalitat de Catalunya for his sabbatical fellowship (2010 PIV 00096). J.A. is a Royal Swedish Academy of Sciences Research Fellow supported by a grant from the Knut and Alice Wallenberg Foundation.

${ }^{1}$ G. C. Hadjipanayis, J. Magn. Magn. Mater. 200, 373 (1999).

${ }^{2}$ T. S. Chin, J. Magn. Magn. Mater. 209, 75 (2000).

${ }^{3}$ D. Weller and A. Moser, IEEE Trans. Magn. 35, 4423 (1999).

${ }^{4}$ S. Gider, B. U. Runge, A. C. Marley, and S. S. P. Parkin, Science 281, 797 (1998).

${ }^{5}$ D. Weller, A. Moser, L. Folks, M. E. Best, W. Lee, M. F. Toney, M. Schwickert, J. U. Thiele, and M. F. Doerner, IEEE Trans. Magn. 36, 10 (2000).

${ }^{6}$ M. Popovici, M. Gich, D. Niznansky, A. Roig, C. Savii, L. Casas, E. Molins, K. Zaveta, C. Enache, J. Sort, S. de Brion, G. Chouteau, and J. Nogués, Chem. Mater. 16, 5542 (2004).

${ }^{7}$ Q. Q. Fang, R. Y. Fang, S. Zhang, and D. S. Dai, J. Appl. Phys. 86, 3878 (1999).

${ }^{8}$ H. Niida, T. Hori, H. Onodera, Y. Yamaguchi, and Y. Nakagawa, J. Appl. Phys. 79, 5946 (1996).

${ }^{9}$ B. Balke, G. H. Fecher, J. Winterlik, and C. Felser, Appl. Phys. Lett. 90, 152504 (2007).

${ }^{10}$ H. Kurt, K. Rode, M. Venkatesan, P. Stamenov, and J. M. D. Coey, Phys. Rev. B 83, 020405R (2011).

${ }^{11}$ S. Mizukami, F. Wu, A. Sakuma, J. Walowski, D. Watanabe, T. Kubota, X. Zhang, H. Naganuma, M. Oogane, Y. Ando, and T. Miyazaki, Phys. Rev. Lett. 106, 117201 (2011).

${ }^{12}$ J.-G. Zhu, Proc. IEEE 96, 1786 (2008).

${ }^{13}$ J. A. Katine and E. E. Fullerton, J. Magn. Magn. Mater. 320, 1217 (2008).

${ }^{14}$ J. Winterlik, B. Balke, G. H. Fecher, C. Felser, M. C. M. Alves, F. Bernardi, and J. Morais, Phys. Rev. B 77, 054406 (2008).

${ }^{15}$ F. Wu, S. Mizukami, D. Watanabe, H. Naganuma, M. Oogane, Y. Ando, and T. Miyazaki, Appl. Phys. Lett. 94, 122503 (2009).

${ }^{16}$ F. Wu, E. P. Sajitha, S. Mizukami, D. Watanabe, T. Miyazaki, H. Naganuma, M. Oogane, and Y. Ando, Appl. Phys. Lett. 96, 042505 (2010).

${ }^{17}$ C. L. Zha, R. K. Dumas, J. Persson, S. M. Mohseni, J. Nogués, and J. Åkerman, IEEE Magn. Lett. 1, 2500104 (2010).

${ }^{18}$ J. Rodriguez-Carvajal, Physica B 192, 55 (1993).

${ }^{19}$ J. G. Moreira, J. K. L. da Silva, and S. O. Kamphorst, J. Phys. A 27, 8079 (1994).

${ }^{20}$ The roughness exponent was calculated as $\alpha=3-\mathrm{D}$, where $\mathrm{D}$ is the fractal dimension. D was obtained using the Nanotech SPM software. I. Horcas, R. Fernandez, J. M. Gomez-Rodriguez, J. Colchero, J. Gomez-Herrero, and A. M. Baro, Rev. Sci. Instrum. 78, 013705 (2007).

${ }^{21}$ K. H. Müller, J. Appl. Phys. 58, 2573 (1985).

${ }^{22}$ S. I. Smirnov and S. V. Komogortsev, J. Magn. Magn. Mater. 320, 1123 (2008).

${ }^{23}$ T. Shima, K. Takanashi, Y. K. Takahashi, and K. Hono, Appl. Phys. Lett. 85, 2571 (2004).

${ }^{24}$ G. Q. Li, H. Takahoshi, H. Ito, H. Saito, S. Ishio, T. Shima, and K. Takanashi, J. Appl. Phys. 94, 5672 (2003).

${ }^{25}$ J. I. Martín, J. Nogués, K. Liu, J. L. Vicent, and I. K. Schuller, J. Magn. Magn. Mater. 256, 449 (2003).

${ }^{26}$ Y. K. Takahashi, T. O. Seki, K. Hono, T. Shima, and K. Takanashi, J. Appl. Phys. 96, 475 (2004). 